\section{Óbitos infantis evitáveis nas coortes de nascimentos de Pelotas, Rio Grande do Sul, Brasil, de 1993 e 2004}

\author{
Avoidable infant deaths in the 1993 and 2004 \\ Pelotas birth cohorts, Rio Grande do \\ Sul State, Brazil \\ Muertes infantiles evitables en las cohortes \\ de nacimiento de 1993 y 2004 de Pelotas, \\ Río Grande do Sul, Brasil
}

\begin{abstract}
Infant mortality classified as avoidable (through immunization, adequate prenatal, childbirth, and neonatal care, adequate diagnostic and therapeutic measures, and adequate health promotion associated with appropriate health care) was compared in the 1993 and 2004 Pelotas Birth Cohorts, Rio Grande do Sul State, Brazil. Deaths were monitored by visits to hospitals, notary public offices, cemeteries, and the Regional Health Division and by a search in the Mortality Information System database. There were 5,249 live births and 111 infant deaths in the $1993 \mathrm{co}$ hort and 4,231 live births and 82 infant deaths in 2004. The avoidable infant mortality rate was 15.2:1,000 live births in 1993 and 15.4 in 2004. Avoidable neonatal and post-neonatal mortality rates were 11.2 and 4.0, respectively, in 1993, and 10.9 and 4.5 in 2004. Preterm births were the main variable associated with avoidable mortality in both cohorts. Strategies to prevent preterm birth may help reduce infant mortality in this context.
\end{abstract}

Infant Mortality; Cause of Death; Cohort Studies
Iná S. Santos 1

Alicia Matijasevich 1

Luís Ramon M. R. Gorgot 1

Neiva C. J. Valle 1

Ana M. Menezes ${ }^{1}$

\section{Resumen}

Comparou-se a mortalidade infantil por causas evitáveis (óbitos reduzíveis por ações de imunoprevenção; por adequada atenção à mulher na gestação e parto e ao recém-nascido; por ações adequadas de diagnóstico e tratamento; e por ações adequadas de promoção à saúde vinculadas às ações adequadas de atenção à saúde) nas coortes de nascimentos de Pelotas, Rio Grande do Sul, Brasil, de 1993 e 2004. Os óbitos foram monitorizados mediante visitas aos hospitais, cartórios, cemitérios, Delegacia Regional de Saúde e rastreio à base de dados do Sistema de Informações sobre Mortalidade (SIM) do Rio Grande do Sul. Na coorte de 1993, houve 5.249 nascidos vivos e 111 óbitos infantis; na de 2004, 4.231 nascidos vivos e 82 óbitos infantis. O coeficiente de mortalidade infantil evitável foi 15,2:1.000 nascidos vivos em 1993 e 15,4 em 2004. Os coeficientes de mortalidades neonatal e pós-neonatal evitáveis foram, respectivamente, 11,2 e 4,0 em 1993 e 10,9 e 4,5 em 2004. Estratégias que visem à prevenção da prematuridade poderão ajudar a reduzir a mortalidade infantil em nosso meio.

Mortalidade Infantil; Causas de Morte; Estudos de Coortes 


\section{Introdução}

Projeções de mortalidade indicam que, até 2015, o Brasil deverá atender ao quarto dos Objetivos de Desenvolvimento do Milênio, traçados pelo Programa das Nações Unidas para o Desenvolvimento: redução em dois terços da mortalidade de menores de cinco anos registrada em torno do ano de 1990 1. Em nível global, a redução da mortalidade entre menores de cinco anos, no período de 2000 a 2010, deveu-se à diminuição nas taxas de óbitos por pneumonia, sarampo e diarreia, enquanto a diminuição das mortes neonatais por causas associadas à prematuridade $\mathrm{e}$ complicações intraparto foi mínima 2.

No Brasil, desde o ano 2000, os coeficientes de mortalidade dos menores de um ano de idade (mortalidade infantil) vêm apresentando padrão de queda constante, passando de 26,1 óbitos por mil nascidos vivos em 2000 , para 15,3 por mil nascidos vivos em $2011^{3}$. Em 2011, a prematuridade, complicações intraparto e infecções perinatais foram as principais causas de mortes neonatais, ao passo que as infecções (influenza e pneumonia), juntamente com as malformações congênitas, responderam pelos óbitos pós-neonatais ${ }^{3}$. No entanto, desigualdades ainda persistem no país, com taxas mais elevadas de óbitos infantis nas regiões Norte (19,9 por mil nascidos vivos) e Nordeste (18 por mil nascidos vivos) em 20113.

Em Pelotas, Rio Grande do Sul, as três coortes de nascimentos mostraram que o coeficiente de mortalidade infantil caiu de 36,4 por mil nascidos vivos em 1982, para 21,1 em 1993 e 19,4 em 2004, uma redução de $42 \%$ entre as duas primeiras coortes $(\mathrm{p}<0,001)$ e de apenas $8 \%$ entre as duas últimas $(\mathrm{p}=0,545)^{4}$. As principais causas de mortalidade infantil na coorte de 2004 foram as perinatais e as infecções respiratórias, muitas das quais seriam evitáveis com os recursos disponíveis no Sistema Único de Saúde (SUS) 5,6. Como os óbitos infantis da coorte de 2004 ocorreram em um período de franca redução da mortalidade infantil no país, o presente estudo teve como objetivos avaliar se houve mudança no coeficiente de mortalidade infantil evitável e comparar seus determinantes entre as crianças das coortes de nascimentos de Pelotas de 1993 e 2004.

\section{Metodologia}

Em 1993 e 2004, cerca de 99\% de todos os nascimentos hospitalares ocorridos em Pelotas de 1o de janeiro a 31 de dezembro dos referidos anos foram acompanhados, dando origem às coortes infantis. A metodologia empregada nas duas co- ortes e nos subestudos da mortalidade infantil foram semelhantes e estão disponíveis 7. Foram monitorizados todos os óbitos infantis ocorridos no período de 1o de janeiro do ano da coorte a 31 de dezembro do ano seguinte, quando a última criança nascida no estudo completava um ano de idade.

Nas duas coortes, foram incluídos os óbitos neonatais precoces (ocorridos nos primeiros sete dias de vida), os neonatais tardios (ocorridos do oitavo até antes de completar o 28o. dia de vida) e os pós-neonatais (de 28 até 364 dias de vida). Os óbitos foram monitorizados mediante idas regulares aos principais hospitais da cidade, onde eram visitadas as unidades de tratamento intensivo, berçários, enfermarias pediátricas e serviços de pronto-socorro. Para detectar óbitos extra-hospitalares, foram visitados os cartórios, os cemitérios e a Delegacia Regional de Saúde. A base de dados do Sistema de Informações sobre Mortalidade (SIM) do Estado do Rio Grande do Sul foi também rastreada em busca de mortes que houvessem ocorrido fora da cidade de Pelotas.

A causa básica do óbito foi investigada através de entrevista com o pediatra responsável. No caso de crianças hospitalizadas, foi realizada uma revisão sistemática do prontuário hospitalar, sendo colhidas informações sobre motivo da internação, história e evolução da doença, exames complementares realizados, tratamento e diagnóstico. Se necessário, as informações contidas no questionário perinatal aplicado à mãe, logo após o nascimento, eram também utilizadas.

Para crianças falecidas entre sete e 364 dias de vida, realizou-se entrevista domiciliar com a mãe, quando era investigada a história clínica da doença e seus antecedentes. Para essa entrevista, foram empregados os questionários da Investigação Interamericana de Mortalidade na Infância 8 , adaptados para a realidade local. Para os óbitos ocorridos fora do hospital ou em outras cidades, as informações foram coletadas dos atestados de óbito, complementadas por entrevista domiciliar com familiares. Em cada coorte, dois pediatras independentes foram responsáveis pela determinação da causa básica do óbito por meio de revisão cuidadosa de toda a informação disponível. Em caso de discordância, um terceiro pediatra era convidado a discutir o caso para uma decisão final. Os atestados foram codificados conforme as $9 \underline{a}$ e $10 \underline{a}$ revisões da Classificação Internacional de Doenças (CID-9 e CID-10) 9,10.

Para a presente análise, os óbitos das duas coortes foram classificados quanto à evitabilidade 11, em quatro grupos: reduzíveis por ações de imunoprevenção; reduzíveis por adequada atenção à mulher na gestação e parto e ao 
recém-nascido; reduzíveis por ações adequadas de diagnóstico e tratamento; e reduzíveis por ações adequadas de promoção à saúde, vinculadas às ações adequadas de atenção à saúde.

De acordo com essa classificação, as causas reduzíveis por adequada atenção à mulher na gestação e parto e ao recém-nascido subdividem-se ainda em 11 reduzíveis por adequada atenção à mulher na gestação (como sífilis congênita, doenças causadas pelo vírus da imunodeficiência humana, afecções maternas que afetam o feto ou o recém-nascido, não obrigatoriamente relacionadas com a gestação em curso, sendo transmitidas pela placenta ou leite materno, complicações maternas da gravidez que afetam o feto ou o recém-nascido, crescimento fetal retardado e desnutrição fetal, transtornos relacionados com gestação de curta duração e baixo peso ao nascer etc.); reduzíveis por adequada atenção à mulher no parto (como outras complicações do trabalho de parto ou do parto que afetam o recém-nascido, transtornos relacionados com gestação prolongada e peso elevado ao nascer, traumatismos de parto, hipóxia intra-uterina e asfixia ao nascer, aspiração neonatal etc.); e reduzíveis por adequada atenção ao recém-nascido (como pneumonia congênita, desconforto respiratório do recém-nascido, hemorragia pulmonar originada no período perinatal, septicemia bacteriana do recém-nascido, onfalite do recémnascido etc.).

Para cada coorte, as causas básicas de óbito foram revisadas por três pediatras, os quais, separadamente, analisaram e classificaram cada morte quanto à evitabilidade. As classificações foram posteriormente comparadas e as diferenças discutidas até o consenso.

Como variáveis independentes, foram utilizadas informações obtidas na fase perinatal de cada uma das coortes, quando foram investigadas características familiares, maternas, assistenciais e do recém-nascido. Para fins de comparabilidade, essas informações foram coletadas de forma semelhante nas duas coortes. A renda familiar foi definida como a soma de todos os valores percebidos por todos os moradores do domicílio no mês anterior à entrevista, sendo posteriormente categorizada em salários mínimos vigentes à época da coleta dos dados $(\leq 1,0 ; 1,1-3,0 ; 3,1-6,0$; 6,1-10,0; > 10,0 salários mínimos) para facilitar a comparação entre coortes. A escolaridade materna corresponde aos anos de educação formal cursados com aprovação.

Dentre as variáveis demográficas maternas, investigaram-se a idade em anos (posteriormente categorizada em <20, 20-34 e >34 anos); a cor da pele, que, na coorte de 2004 , foi autorreferida pela mãe como branca, preta, morena ou parda, amarela ou asiática e indígena, sendo, posteriormente, dicotomizada em branca e preta/outra, para comparabilidade com a coorte de 1993, que havia coletado essa informação em três categorias (branca, preta e outra); e a situação conjugal dicotomizada em "casada/com companheiro" ou "sem companheiro", independentemente do estado civil.

O peso pré-gestacional foi relatado pela mãe no perinatal. Na coorte de 1993, a altura materna foi aferida ainda no hospital do parto (no estudo perinatal) e, na de 2004, na primeira visita de acompanhamento no domicílio das crianças, aos três meses de idade. Em ambas as coortes, para aferição da altura, empregou-se estadiômetro de metal confeccionado especialmente para a pesquisa. O índice de massa corporal (IMC) pré-gestacional foi calculado como o peso em quilos dividido pelo quadrado da altura em metros, sendo categorizado em quatro grupos para as análises $\left(<18,5 ; 18,5-24,9 ; 25,0-29,9 ; \geq 30 \mathrm{~kg} / \mathrm{m}^{2}\right)$.

O número de recém-nascidos vivos ou mortos, anteriores à criança da coorte, foi categorizado em 0,1 e $\geq 2$. O tabagismo materno foi definido como o consumo de pelo menos um cigarro por dia em qualquer dos trimestres da gestação. Adicionalmente, investigou-se se a gestação havia sido planejada (sim ou não).

Dentre as variáveis assistenciais, investigaram-se o tipo de financiamento do parto (SUS ou particular/convênios); o trimestre de início e o número de consultas pré-natais; realização de vacinação antitetânica (sim ou não); diagnóstico médico de anemia, hipertensão arterial, diabete mellitus e infecção do trato urinário durante a gestação (sim ou não); tipo de parto (vaginal ou cesariana); parto assistido por médico (sim ou não); e recém-nascido assistido por pediatra na sala de parto (sim ou não).

Entre as características das crianças, além do sexo, coletou-se o peso de nascimento e calculou-se a idade gestacional ao nascer. Os recémnascidos com peso inferior a $2.500 \mathrm{~g}$ foram classificados como com baixo peso ao nascer. A idade gestacional foi calculada com base no algoritmo proposto pelo National Center for Health Statistics (NCHS) 12, usando-se a data da última menstruação sempre que consistente com peso, comprimento e circunferência da cabeça ao nascer, conforme as curvas normais para esses parâmetros para cada semana de idade gestacional 13. Quando a data da última menstruação era desconhecida ou inconsistente, empregouse a idade estimada pelo método de Dubowitz et al. ${ }^{14}$, que foi realizado em todos os recémnascidos.

Os nascimentos com menos de 37 semanas de gestação foram classificados como pré- 
termos. A presença de restrição do crescimento intrauterino foi definida pela comparação com os valores da curva de Williams et al. 15. Foram classificadas como pequenas para a idade gestacional as crianças que estavam abaixo do percentil 10 do peso para a idade gestacional e sexo, e como grandes para a idade gestacional (GIG) aquelas acima do percentil 90. O escore de Apgar no 5o minuto foi dicotomizado em $\geq 7$ e $<7$. A ocorrência de complicações neonatais foi registrada, sendo a variável posteriormente dicotomizada para análise em sim ou não.

Os coeficientes de mortalidades neonatal, pós-neonatal e infantil evitáveis das duas coortes foram calculados e comparados. Os nascimentos com idade gestacional desconhecida foram excluídos apenas das análises específicas por idade gestacional. Foram calculadas a incidência e a razão de incidências cumulativas para cada coorte separadamente, conforme as variáveis independentes. As razões de incidências cumulativas brutas e ajustadas foram obtidas por regressão logística, seguindo um modelo hierárquico construído pelos autores 16 , com quatro níveis de determinação (primeiro nível: variáveis socioeconômicas; segundo nível: características demográficas e reprodutivas maternas e tabagismo na gravidez; terceiro nível: características do parto e do pré-natal e intercorrências na gravidez; quarto nível: características da criança ao nascer e complicações neonatais). As variáveis associadas ao óbito evitável com valor de $\mathrm{p} \leq 0,20$ foram mantidas na análise ajustada, para ajuste das demais. As análises foram realizadas com o pacote Stata versão 11 (Stata Corp., College Station, Estados Unidos). Antes das análises ajustadas, os bancos das duas coortes foram unificados e reali- zados testes de interação entre a variável "ano de nascimento (1993 e 2004)” e as demais variáveis independentes. Embora não tenham sido identificadas interações estatisticamente significativas, as análises controladas foram realizadas e apresentadas para cada coorte separadamente, a fim de se compararem os determinantes de mortalidade de uma coorte com os da outra.

O protocolo do estudo foi aprovado pelo Comitê de Ética da Faculdade de Medicina da Universidade Federal de Pelotas. Em 1993, foi obtido consentimento verbal das mães para participar no estudo. Em 2004, solicitou-se também o consentimento por escrito.

\section{Resultados}

O coeficiente de mortalidade infantil por mil nascidos vivos segundo causas evitáveis foi de 15,2, em 1993, e de 15,4 ( $p=0,933)$, em 2004. Os coeficientes de mortalidade neonatal e pós-neonatal por mil nascidos vivos segundo causas evitáveis foram, respectivamente, de 11,2 e 4,0, em 1993, e de 10,9 e 4,5, em 2004.

A Tabela 1 apresenta o número absoluto de óbitos e seu percentual entre todas as mortes de menores de um ano, por grupos de causas evitáveis, separadamente para cada coorte. Em nenhuma das coortes foram verificados óbitos por doenças preveníveis por imunização. Não se observou diferença estatisticamente significativa entre as coortes quanto aos óbitos por cada um dos grupos de causas evitáveis, mas, entre 1993 e 2004, houve diminuição nas mortes decorrentes de causas reduzíveis por adequada atenção ao recém-nascido (de $7,2 \%$ para $3,7 \%$ ) e por ações

Tabela 1

Número de óbitos infantis por causas evitáveis nas coortes de nascimentos de Pelotas, Rio Grande do Sul, Brasil, de 1993 e 2004.

\begin{tabular}{|c|c|c|c|}
\hline \multirow[t]{2}{*}{ Classificação } & 1993 & 2004 & \multirow[t]{2}{*}{ Valor de $p$ * } \\
\hline & n (\%) & n (\%) & \\
\hline Reduzíveis por ações de imunoprevenção & 0 & 0 & - \\
\hline \multicolumn{4}{|c|}{ Reduzíveis por adequada atenção à mulher na gestação e parto e ao recém-nascido } \\
\hline Reduzíveis por adequada atenção à mulher na gestação & $41(36,9)$ & $41(50,0)$ & 0,070 \\
\hline Reduzíveis por adequada atenção à mulher no parto & $6(5,4)$ & $4(4,9)$ & 0,870 \\
\hline Reduzíveis por adequada atenção ao recém-nascido & $8(7,2)$ & $3(3,7)$ & 0,293 \\
\hline Reduzíveis por ações adequadas de diagnóstico e tratamento & $8(7,2)$ & $10(12,2)$ & 0,239 \\
\hline Reduzíveis por ações adequadas de promoção à saúde & $17(15,3)$ & $7(8,5)$ & 0,158 \\
\hline Demais causas não evitáveis (malformações congênitas) & $26(23,4)$ & $13(15,9)$ & 0,195 \\
\hline Mal definidas & $5(4,5)$ & $4(4,9)$ & 0,903 \\
\hline Total & $111(100,0)$ & $82(100,0)$ & - \\
\hline
\end{tabular}


adequadas de promoção à saúde (de 15,3\% para 8,5\%). Já a proporção de óbitos preveníveis por adequada atenção à mulher na gestação e por ações adequadas de diagnóstico e tratamento aumentou, respectivamente, de $36,9 \%$ para $50 \%$ e de $7,2 \%$ para $12,2 \%$.

As Tabelas 2 e 3 mostram que, entre 1993 e 2004, ocorreram mudanças estatisticamente significativas na distribuição de determinantes sociais (renda familiar, escolaridade materna e situação conjugal), biológicos (idade, cor, altura, IMC pré-gestacional e número de recémnascidos vivos ou mortos, anteriores à criança da coorte,), comportamentais (tabagismo e planejamento da gravidez) e de morbidade materna (prevalências de anemia, hipertensão arterial e infecção urinária). O mesmo se deu na utilização de serviços de saúde (idade gestacional de início e número de consultas pré-natais, cobertura vacinal contra o tétano, tipo e modo de financiamento do parto e presença de pediatra na sala de parto) e nas características do recém-nascido (sexo, idade gestacional, crescimento intrauterino e prevalência de complicações neonatais).
Nas duas coortes (Tabela 2), a incidência de óbitos evitáveis no primeiro ano de vida foi maior entre as crianças de famílias com menor renda (risco cerca de nove vezes maior em 1993 e seis vezes maior em 2004); filhos de mães com menor escolaridade (cinco e 11 vezes maior, respectivamente, em 1993 e 2004, entre os filhos de mães com zero a quatro anos de escolaridade, em comparação aos de mães com mais de 11 anos de educação formal); com cor de pele preta/outra, em comparação às brancas (risco $84 \%$ maior em 1993 e duas vezes maior em 2004); que não fizeram acompanhamento pré-natal (risco 6,6 e 18,7 vezes maior do que o dos filhos de mães que consultaram sete vezes ou mais) ou que consultaram de uma a três vezes durante a gestação (7,5 e 11 vezes maior, respectivamente, em 1993 e 2004 em comparação aos de mães que consultaram sete vezes ou mais); e cujo parto foi financiado pelo SUS (cinco e 2,8 vezes maior em 1993 e 2004, respectivamente, em comparação aos particulares e mediante convênios).

Tabela 2

Incidência de mortes evitáveis entre os nascidos vivos das coortes de nascimentos de Pelotas, Rio Grande do Sul, Brasil, de 1993 (N = 5.249 ) e 2004 ( $N=4.231)$, de acordo com características maternas

\begin{tabular}{|c|c|c|c|c|c|c|c|c|c|}
\hline \multirow[t]{2}{*}{ Características } & \multicolumn{4}{|c|}{1993} & \multicolumn{4}{|c|}{2004} & \multirow{2}{*}{$\begin{array}{l}\text { Valor } \\
\text { de } p \text { * }\end{array}$} \\
\hline & n (\%) & $\begin{array}{c}\text { Incidência } \\
\text { n (\%) }\end{array}$ & $\begin{array}{l}\text { Risco relativo } \\
\text { (IC95\%) }\end{array}$ & $\begin{array}{l}\text { Valor } \\
\text { de } p\end{array}$ & n (\%) & $\begin{array}{c}\text { Incidência } \\
\text { n (\%) }\end{array}$ & $\begin{array}{l}\text { Risco relativo } \\
\text { (IC95\%) }\end{array}$ & $\begin{array}{l}\text { Valor } \\
\text { de p }\end{array}$ & \\
\hline Renda familiar (salários & & & & 0,008 & & & & 0,095 & $<0,001$ \\
\hline \multicolumn{10}{|l|}{ mínimos) ** } \\
\hline$\leq 1,0$ & $967(18,4)$ & $24(2,4)$ & $9,56(1,30 ; 70,40)$ & & $884(20,9)$ & $22(2,5)$ & $6,05(0,82 ; 44,65)$ & & \\
\hline $1,1-3,0$ & $2.260(43,1)$ & $41(1,8)$ & $6,98(0,96 ; 50,63)$ & & $1.939(45,9)$ & $29(1,5)$ & $3,63(0,50 ; 26,56)$ & & \\
\hline $3,1-6,0$ & $1.204(22,9)$ & $11(0,9)$ & $3,52(0,46 ; 27,16)$ & & $945(22,4)$ & $13(1,4)$ & $3,34(0,44 ; 25,43)$ & & \\
\hline $6,1-10,0$ & $433(8,2)$ & $3(0,7)$ & $2,67(0,28 ; 25,54)$ & & $243(5,8)$ & $1(0,4)$ & 1,00 & & \\
\hline$>10,0$ & $385(7,3)$ & $1(0,3)$ & 1,00 & & $207(4,9)$ & $0(0,0)$ & - & & \\
\hline Escolaridade (anos) & & & & 0,011 & & & & 0,018 & $<0,001$ \\
\hline $0-4$ & $1.468(28,0)$ & $35(2,4)$ & $5,09(1,23 ; 21,08)$ & & $655(15,6)$ & $17(2,6)$ & $10,90(1,46 ; 81,62)$ & & \\
\hline $5-8$ & $2.424(46,2)$ & $33(1,4)$ & $2,91(0,70 ; 12,07)$ & & $1.731(41,3)$ & $31(1,8)$ & $7,52(1,03 ; 54,95)$ & & \\
\hline $9-11$ & $923(17,6)$ & $10(1,1)$ & $2,31(0,51 ; 10,51)$ & & $1.382(33,0)$ & $15(1,1)$ & $4,56(0,60 ; 34,42)$ & & \\
\hline$>11$ & $427(8,1)$ & $2(0,5)$ & 1,00 & & $420(10,0)$ & $1(0,2)$ & 1,00 & & \\
\hline Situação conjugal & & & & 0,288 & & & & 0,001 & $<0,001$ \\
\hline $\begin{array}{l}\text { Casada/Com } \\
\text { companheiro }\end{array}$ & $4.600(87,6)$ & $67(1,5)$ & 1,00 & & $3.536(83,6)$ & $44(1,2)$ & 1,00 & & \\
\hline Sem companheiro & $649(12,4)$ & $13(2,0)$ & $1,38(0,76 ; 2,48)$ & & $695(16,4)$ & $21(3,0)$ & $2,43(1,45 ; 4,06)$ & & \\
\hline Idade (anos) & & & & 0,085 & & & & 0,27 & $<0,001$ \\
\hline$<20$ & $915(17,4)$ & $12(1,3)$ & $0,93(0,50 ; 1,73)$ & & $800(18,9)$ & $16(2,0)$ & $1,30(0,74 ; 2,30)$ & & \\
\hline $20-34$ & $3.756(71,6)$ & $53(1,4)$ & 1,00 & & $2.866(67,8)$ & $44(1,5)$ & 1,00 & & \\
\hline$>34$ & $577(11,0)$ & $15(2,6)$ & $1,84(1,05 ; 3,25)$ & & $563(13,3)$ & $5(0,9)$ & $0,58(0,23 ; 1,45)$ & & \\
\hline
\end{tabular}

(continua) 
Tabela 2 (continuação)

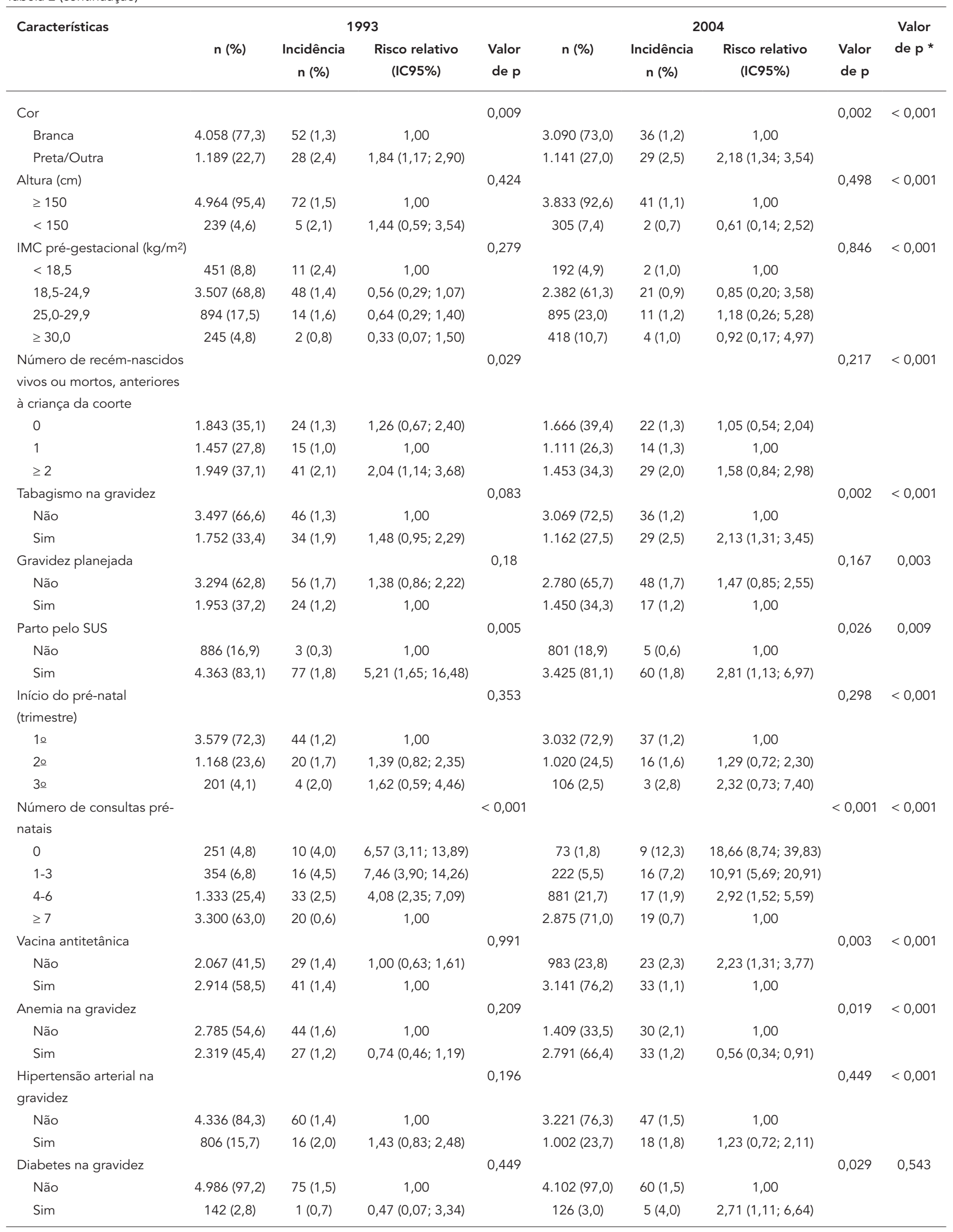

(continua) 
Tabela 2 (continuação)

\begin{tabular}{|c|c|c|c|c|c|c|c|c|c|}
\hline \multirow[t]{2}{*}{ Características } & \multicolumn{4}{|c|}{1993} & \multicolumn{4}{|c|}{2004} & \multirow{2}{*}{$\begin{array}{l}\text { Valor } \\
\text { de } p \text { * }\end{array}$} \\
\hline & n (\%) & $\begin{array}{c}\text { Incidência } \\
\text { n (\%) }\end{array}$ & $\begin{array}{l}\text { Risco relativo } \\
\text { (IC95\%) }\end{array}$ & $\begin{array}{l}\text { Valor } \\
\text { de p }\end{array}$ & n (\%) & $\begin{array}{c}\text { Incidência } \\
\text { n (\%) }\end{array}$ & $\begin{array}{l}\text { Risco relativo } \\
\text { (IC95\%) }\end{array}$ & $\begin{array}{l}\text { Valor } \\
\text { de } p\end{array}$ & \\
\hline Diabetes na gravidez & & & & 0,449 & & & & 0,029 & 0,543 \\
\hline Não & $4.986(97,2)$ & $75(1,5)$ & 1,00 & & $4.102(97,0)$ & $60(1,5)$ & 1,00 & & \\
\hline $\operatorname{Sim}$ & $142(2,8)$ & $1(0,7)$ & $0,47(0,07 ; 3,34)$ & & $126(3,0)$ & $5(4,0)$ & $2,71(1,11 ; 6,64)$ & & \\
\hline $\begin{array}{l}\text { Infecção urinária na } \\
\text { gravidez }\end{array}$ & & & & 0,013 & & & & 0,407 & $<0,001$ \\
\hline Não & $3.413(66,4)$ & $39(1,1)$ & 1,00 & & $2.648(62,8)$ & $37(1,4)$ & 1,00 & & \\
\hline Sim & $1.726(33,6)$ & $35(2,0)$ & $1,77(1,13 ; 2,79)$ & & $1.569(37,2)$ & $27(1,7)$ & $1,23(0,75 ; 2,01)$ & & \\
\hline Tipo de parto & & & & 0,555 & & & & 0,168 & $<0,001$ \\
\hline Vaginal & $3.647(69,5)$ & $58(1,6)$ & 1,00 & & $2.309(54,6)$ & $41(1,8)$ & 1,00 & & \\
\hline Cesariana & $1.602(30,5)$ & $22(1,4)$ & $0,86(0,53 ; 1,41)$ & & $1.922(45,4)$ & $24(1,3)$ & $0,70(0,43 ; 1,16)$ & & \\
\hline Parto atendido por médico & & & & 0,899 & & & & 0,229 & 0,157 \\
\hline Não & $607(11,7)$ & $9(1,5)$ & $0,96(0,48 ; 1,90)$ & & $453(10,8)$ & $10(2,2)$ & $1,51(0,77 ; 2,93)$ & & \\
\hline Sim & $4.580(88,3)$ & $71(1,6)$ & 1,00 & & $3.752(89,2)$ & $55(1,5)$ & 1,00 & & \\
\hline Pediatra na sala de parto & & & & 0,112 & & & & 0,966 & $<0,001$ \\
\hline Não & $2.503(53,1)$ & $31(1,2)$ & $0,69(0,43 ; 1,09)$ & & $749(21,7)$ & $9(1,2)$ & $0,98(0,47 ; 2,05)$ & & \\
\hline Sim & $2.214(46,9)$ & $40(1,8)$ & 1,00 & & $2.703(78,3)$ & $33(1,2)$ & 1,00 & & \\
\hline
\end{tabular}

* Valor de p da diferença entre coorte de 1993 e 2004.

** Salários mínimos vigentes no mês de nascimento da criança.

Na Tabela 3, a incidência de óbitos evitáveis foi 15 vezes mais frequente, em ambas as coortes, entre os nascidos com baixo peso, em comparação aos nascidos com 2.500g ou mais, e aumentou com a diminuição da idade gestacional ao nascer. Entre os pré-termos limítrofes (34-36 semanas de idade gestacional), os óbitos evitáveis foram cerca de três vezes mais frequentes do que entre os nascidos a termo. O Apgar $<7$ no quinto minuto associou-se a uma incidência 34 vezes maior de óbitos evitáveis em 1993 e 17 vezes maior em 2004, associando-se ainda à ocorrência de complicações neonatais, com incidência 26 e 16 vezes maior, respectivamente, em 1993 e 2004 (Tabela 3).

O sexo do recém-nascido, a maior paridade materna e a história de infecção do trato urinário na gestação associaram-se ao maior risco de morte entre as crianças em 1993, mas não foram estatisticamente significativos em 2004. Em 1993, o risco entre os meninos era $53 \%$ maior do que o das meninas (Tabela 3); os filhos de mães com dois ou mais partos anteriores tinham risco duas vezes maior do que os primogênitos (Tabela 2); e os filhos de mães com história de infecção urinária na gestação tinham $77 \%$ mais risco de morrer, em comparação aos filhos de mães que não relataram infecção urinária (Tabela 2).

Em 1993, características como serem filhos de mães que viviam sem companheiro, fuman- tes, com história de diabetes e que não receberam imunização antitetânica na gestação não se associaram ao risco de morrer; entretanto, em 2004, apresentaram tal associação (Tabela 2). O risco de morrer entre os filhos de mães solteiras, fumantes, diabéticas na gestação ou que não receberam vacina antitetânica foi duas vezes maior que entre suas contrapartes.

A Tabela 4 apresenta o odds ratio (OR) para mortalidade infantil na coorte de 1993, de acordo com o modelo final da análise multivariável. A maioria dos achados observados na análise bruta confirmou-se na ajustada. Foram estatisticamente significativas as associações com as seguintes variáveis: menor renda familiar mensal em salários mínimos, cor da pele preta/outra, idade materna maior de 34 anos, menor número de visitas pré-natais, infecção urinária na gestação e prematuridade. Entre as crianças que não foram assistidas por pediatras na sala de parto, $o$ OR para morrer por causa evitável foi $55 \%$ menor, em comparação com as que contaram com esse recurso ao nascer.

Em 2004 (Tabela 5), as variáveis maternas que permaneceram estatisticamente associadas ao risco de morrer por causa evitável foram baixa escolaridade, ausência de companheiro, cor da pele preta/outra, tabagismo durante a gestação, menor número de visitas pré-natais e história de diabetes na gestação. Dentre as características 
Tabela 3

Incidência de mortes evitáveis entre os nascidos vivos das coortes de nascimentos de Pelotas, Rio Grande do Sul, Brasil, de 1993 (N = 5.249 ) e 2004

( $N=4.231)$, de acordo com as características da criança ao nascer.

\begin{tabular}{|c|c|c|c|c|c|c|c|c|c|}
\hline \multirow[t]{2}{*}{ Características } & \multicolumn{4}{|c|}{1993} & \multicolumn{4}{|c|}{2004} & \multirow{2}{*}{$\begin{array}{l}\text { Valor } \\
\text { de } p \text { * }\end{array}$} \\
\hline & $\mathbf{n}$ & $\begin{array}{c}\text { Incidência } \\
\text { n (\%) }\end{array}$ & $\begin{array}{l}\text { Risco relativo } \\
\text { (IC95\%) }\end{array}$ & $\begin{array}{l}\text { Valor } \\
\text { de } p\end{array}$ & $\mathbf{n}$ & $\begin{array}{c}\text { Incidência } \\
\text { n (\%) }\end{array}$ & $\begin{array}{l}\text { Risco relativo } \\
\text { (IC95\%) }\end{array}$ & $\begin{array}{l}\text { Valor } \\
\text { de } p\end{array}$ & \\
\hline Sexo & & & & 0,007 & & & & 0,752 & 0,008 \\
\hline Feminino & $2.667(50,8)$ & $28(1,1)$ & $0,53(0,34 ; 0,84)$ & & $2.035(48,1)$ & $30(1,5)$ & $0,92(0,57 ; 1,50)$ & & \\
\hline Masculino & $2.580(49,2)$ & $51(2,0)$ & 1,00 & & $2.196(51,9)$ & $35(1,6)$ & 1,00 & & \\
\hline Baixo peso ao nascer & & & & $<0,001$ & & & & $<0,001$ & 0,649 \\
\hline Não & $4.722(90,3)$ & $28(0,6)$ & 1,00 & & $3.804(90,0)$ & $23(0,6)$ & 1,00 & & \\
\hline Sim & $510(9,7)$ & $46(9,1)$ & $15,21(9,59 ; 24,12)$ & & $424(10,0)$ & $41(9,7)$ & $15,99(9,69 ; 26,39)$ & & \\
\hline $\begin{array}{l}\text { Idade gestacional } \\
\text { (semanas) }\end{array}$ & & & & $<0,001$ & & & & $<0,001$ & $<0,001$ \\
\hline$<32$ & $43(0,8)$ & $21(48,8)$ & $93,24(56,39 ; 154,16)$ & & $76(1,8)$ & $28(36,8)$ & $66,39(39,19 ; 112,45)$ & & \\
\hline $32-33$ & $75(1,4)$ & $10(13,3)$ & $25,46(12,62 ; 51,34)$ & & $65(1,5)$ & $4(6,2)$ & $11,09(3,90 ; 31,54)$ & & \\
\hline $34-36$ & $471(9,1)$ & $7(1,5)$ & $2,84(1,23 ; 6,55)$ & & $472(11,2)$ & $7(1,5)$ & $2,67(1,14 ; 6,29)$ & & \\
\hline$\geq 37$ & $4.582(88,6)$ & $24(0,5)$ & 1,00 & & $3.604(85,5)$ & $20(0,6)$ & 1,00 & & \\
\hline $\begin{array}{l}\text { Pequeno para idade } \\
\text { gestacional }\end{array}$ & & & & 0,171 & & & & 0,668 & $<0,001$ \\
\hline Não & $4.677(90,6)$ & $53(1,1)$ & 1,00 & & $3.712(88,0)$ & $53(1,4)$ & 1,00 & & \\
\hline Sim & $487(9,4)$ & $9(1,9)$ & $1,63(0,81 ; 3,29)$ & & $505(12,0)$ & $6(1,2)$ & $0,83(0,36 ; 1,93)$ & & \\
\hline $\begin{array}{l}\text { Grande para idade } \\
\text { gestacional }\end{array}$ & & & & 0,255 & & & & 0,108 & 0,001 \\
\hline Não & $4.802(93,0)$ & $60(1,3)$ & 1,00 & & $3.989(94,6)$ & $53(1,3)$ & 1,00 & & \\
\hline $\operatorname{Sim}$ & $362(7,0)$ & $2(0,6)$ & $0,44(0,11 ; 1,80)$ & & $228(5,4)$ & $6(2,6)$ & $1,98(0,86 ; 4,56)$ & & \\
\hline $\begin{array}{l}\text { Complicações } \\
\text { neonatais }\end{array}$ & & & & $<0,001$ & & & & $<0,001$ & $<0,001$ \\
\hline Não & $4.849(92,5)$ & $25(0,5)$ & 1,00 & & $3.811(90,4)$ & $22(0,6)$ & 1,00 & & \\
\hline Sim & $391(7,5)$ & $52(13,3)$ & $25,80(16,19 ; 41,10)$ & & $403(9,6)$ & $38(9,4)$ & $16,33(9,76 ; 27,34)$ & & \\
\hline Apgar 5o minuto & & & & $<0,001$ & & & & $<0,001$ & 0,142 \\
\hline$\geq 7$ & $3.744(98,3)$ & $28(0,8)$ & 1,00 & & $4.112(97,8)$ & $44(1,1)$ & 1,00 & & \\
\hline$<7$ & $66(1,7)$ & $17(25,8)$ & $34,44(19,84 ; 59,78)$ & & $92(2,2)$ & $17(18,5)$ & $17,27(10,26 ; 29,05)$ & & \\
\hline
\end{tabular}

* Valor de p da diferença entre coorte de 1993 e 2004.

do recém-nascido, mantiveram-se associadas prematuridade, complicações neonatais e Apgar $<7$ no quinto minuto. Crianças da coorte de 2004 que eram filhas de mães com história de anemia durante a gestação apresentaram menor risco de morrer por causa evitável no primeiro ano de vida.

\section{Discussão}

Este estudo apresenta aspectos positivos e limitações. Entre os aspectos positivos incluem-se a coleta primária dos dados de mortalidade no primeiro ano de vida em duas coortes de nascimento, da mesma cidade, com 11 anos de inter- valo entre ambas; a monitorização simultânea de várias fontes de informação de mortalidade; e o fato de a metodologia de coleta das informações, tanto dos óbitos, quanto das variáveis independentes, haverem sido semelhantes nas duas coortes, garantindo a comparabilidade dos desfechos e exposições. As principais limitações referem-se à possibilidade de viés de informação sobre as exposições de interesse, uma vez que a maioria foi obtida por relato da mãe. Ainda, o tempo decorrido desde os óbitos das crianças das duas coortes até o momento em que as análises do presente estudo foram processadas pode ser considerado uma limitação. No entanto, não foi comprovada a hipótese testada de que, diante do cenário de melhora nos indicadores 
Tabela 4

Incidência de mortes evitáveis entre os nascidos vivos da coorte de nascimentos de Pelotas, Rio Grande do Sul, Brasil, de 1993 ( $N=5.249$ ), de acordo com variáveis independentes (análise ajustada).

\begin{tabular}{|c|c|c|c|}
\hline Nível & Características & Risco relativo ajustado & Valor de $p$ \\
\hline \multirow[t]{6}{*}{1} & Renda familiar mensal (salários mínimos) * & & 0,008 \\
\hline & $\leq 1,0$ & $9,56(1,30 ; 70,40)$ & \\
\hline & $1,1-3,0$ & $6,98(0,96 ; 50,63)$ & \\
\hline & $3,1-6,0$ & $3,52(0,46 ; 27,16)$ & \\
\hline & $6,1-10,0$ & $2,67(0,28 ; 25,54)$ & \\
\hline & $>10,0$ & 1,00 & \\
\hline \multirow[t]{7}{*}{2} & Cor da mãe & & 0,05 \\
\hline & Branca & 1,00 & \\
\hline & Preta/Outra & $1,57(1,00 ; 2.45)$ & \\
\hline & Idade materna (anos) & & 0,026 \\
\hline & $<20$ & $0,82(0,44 ; 1,53)$ & \\
\hline & $20-34$ & 1,00 & \\
\hline & $>34$ & $2,04(1,16 ; 3,61)$ & \\
\hline \multirow[t]{14}{*}{3} & Parto pelo SUS & & 0,089 \\
\hline & Não & 1,00 & \\
\hline & Sim & $4,03(0,80 ; 20,05)$ & \\
\hline & Número de visitas pré-natais & & $<0,001$ \\
\hline & 0 & $9,01(3,63 ; 22,35)$ & \\
\hline & $1-3$ & $6,08(2,84 ; 13,00)$ & \\
\hline & $4-6$ & $4,21(2,25 ; 7,89)$ & \\
\hline & $\geq 7$ & 1,00 & \\
\hline & Infecção urinária na gravidez & & 0,014 \\
\hline & Não & 1,00 & \\
\hline & Sim & $1,82(1,13 ; 2,94)$ & \\
\hline & Pediatra na sala de parto & & 0,002 \\
\hline & Não & $0,45(0,28 ; 0,74)$ & \\
\hline & Sim & 1,00 & \\
\hline \multirow[t]{11}{*}{4} & Idade gestacional ao nascer (semanas) & & $<0,001$ \\
\hline & $<32$ & $20,89(4,52 ; 96,58)$ & \\
\hline & $32-33$ & $6,25(1,40 ; 27,89)$ & \\
\hline & $34-36$ & $1,89(0,47 ; 7,63)$ & \\
\hline & $\geq 37$ & 1,00 & \\
\hline & Complicações neonatais & & 0,141 \\
\hline & Não & 1,00 & \\
\hline & $\operatorname{Sim}$ & $2,79(0,71 ; 10,93)$ & \\
\hline & Apgar 5o minuto & & 0,089 \\
\hline & $\geq 7$ & 1,00 & \\
\hline & $<7$ & $1,83(0,91 ; 3,66)$ & \\
\hline
\end{tabular}

SUS: Sistema Único de Saúde.

* Renda familiar mensal em salários mínimos vigentes no mês de nascimento da criança.

regionais e nacionais no período 3 , os resultados em Pelotas revelariam as causas não evitáveis como as principais envolvidas na persistência dos indicadores de mortalidade infantil, enfatizando a reconhecida importância da constante monitorização desse indicador e das causas de óbito nele envolvidas 2 .
Entre as 9.480 crianças nascidas vivas nos anos de 1993 e 2004, na cidade de Pelotas, 193 faleceram antes de completar um ano de idade. Destas, em ambas as coortes, cerca de três quartos (72\% entre as crianças de 1993 e $79 \%$ entre as de 2004) morreram por causas potencialmente evitáveis. Tal resultado indica que o 
Tabela 5

Incidência de mortes evitáveis entre os nascidos vivos da coorte de nascimentos de Pelotas, Rio Grande do Sul, Brasil, de 2004 ( $N=4.231)$, de acordo com variáveis independentes (análise ajustada).

\begin{tabular}{|c|c|c|c|}
\hline Nível & Características & Risco relativo ajustado & Valor de $p$ \\
\hline \multirow[t]{8}{*}{1} & Escolaridade materna (anos) & & 0,042 \\
\hline & $0-4$ & $7,22(0,96 ; 54,46)$ & \\
\hline & $5-8$ & $4,82(0,65 ; 35,65)$ & \\
\hline & $9-11$ & $3,10(0,41 ; 23,58)$ & \\
\hline & $>11$ & 1,00 & \\
\hline & Situação conjugal & & 0,001 \\
\hline & Casada/Com companheiro & 1,00 & \\
\hline & Sem companheiro & $2,33(1,39 ; 3,91)$ & \\
\hline \multirow[t]{6}{*}{2} & Cor da mãe & & 0,04 \\
\hline & Branca & 1,00 & \\
\hline & Preta/Outra & $1,66(1,02 ; 2,71)$ & \\
\hline & Tabagismo na gravidez & & 0,048 \\
\hline & Não & 1,00 & \\
\hline & Sim & $1,65(1,00 ; 2,71)$ & \\
\hline \multirow[t]{14}{*}{3} & Número de visitas pré-natais & & $<0,001$ \\
\hline & 0 & - & \\
\hline & $1-3$ & $5,82(2,58 ; 13,12)$ & \\
\hline & $4-6$ & $1,98(0,97 ; 4,01)$ & \\
\hline & $\geq 7$ & 1,00 & \\
\hline & Vacina antitetânica na gravidez & & 0,088 \\
\hline & Não & $1,66(0,93 ; 2,98)$ & \\
\hline & Sim & 1,00 & \\
\hline & Anemia na gravidez & & 0,049 \\
\hline & Não & 1,00 & \\
\hline & Sim & $0,58(0,33 ; 1,00)$ & \\
\hline & Diabetes na gravidez & & 0,001 \\
\hline & Não & 1,00 & \\
\hline & Sim & $4,68(1,95 ; 11,23)$ & \\
\hline \multirow[t]{11}{*}{4} & Idade gestacional (semanas) & & $<0,001$ \\
\hline & $<32$ & $12,10(4,69 ; 31,25)$ & \\
\hline & $32-33$ & $5,78(1,95 ; 17,13)$ & \\
\hline & $34-36$ & $1,15(0,47 ; 2,85)$ & \\
\hline & $\geq 37$ & 1,00 & \\
\hline & Complicações neonatais & & 0,001 \\
\hline & Não & 1,00 & \\
\hline & Sim & $3,67(1,67 ; 8,03)$ & \\
\hline & Apgar 5o minuto & & 0,033 \\
\hline & $\geq 7$ & 1,00 & \\
\hline & $<7$ & $2,28(1,07 ; 4,85)$ & \\
\hline
\end{tabular}

risco de morrer por causas evitáveis manteve-se praticamente inalterado por mais de uma década em Pelotas, ao contrário do que ocorreu na Região Sul e no país como um todo entre 1997 e $2006{ }^{17}$. Neste período, houve uma redução de $32,4 \%$ no coeficiente de mortes infantis evitáveis no Sul (de 12,8 para 8,64 por mil nascidos vivos) e de $37,3 \%$ no Brasil (de 22,8 para 14,3 por mil nascidos vivos) 17 .

O progresso nos indicadores de mortalidade infantil verificado no Brasil em 2011, com taxas de 15,3 mil nascidos vivos (neonatal precoce de 
8,1 por mil nascidos vivos; neonatal tardia de 2,5 por mil nascidos vivos; e pós-neonatal de 4,7 por mil nascidos vivos) ${ }^{3}$ refletem o efeito de inúmeras intervenções bem-sucedidas, tanto sociais quanto na organização do sistema de saúde, implementadas no país a partir da década de 8018 . Contudo, inequidades sociais decorrentes, principalmente, do nível econômico (renda e escolaridade materna), cor da pele e grupo étnico, com reflexos negativos sobre os indicadores de mortalidade infantil, ainda persistem e se repetem nos dias atuais, até mesmo em países desenvolvidos da Europa e nos Estados Unidos 3,19,20.

Como verificado neste estudo, a população de gestantes das duas coortes eram diferentes quanto a importantes indicadores socioeconômicas, biológicos, comportamentais e de qualidade dos serviços de saúde. Alguns desses indicadores melhoraram entre 1993 e 2004, como aumento da escolaridade materna, diminuição do tabagismo e da prevalência de anemia na gravidez, planejamento da gravidez por maior proporção de mulheres, aumento do número de consultas pré-natais, início do acompanhamento em idade gestacional mais precoce, aumento da cobertura da vacinação antitetânica e presença de pediatra na sala de parto para a maioria dos recém-nascidos. Por outro lado, houve diminuição da renda familiar das gestantes, aumento da proporção de mulheres sem companheiro, adolescentes, com baixa estatura e com sobrepeso ou obesidade, nulíparas e com relato de hipertensão arterial durante a gestação; registrou-se, ainda, aumento da taxa de cesarianas no período.

Embora estatisticamente não significativas, as variações de causas de mortes evitáveis entre 1993 e 2004 em Pelotas apresentaram as mesmas direções das variações observadas no país entre 1996 e 2007: aumento dos óbitos decorrentes de causas reduzíveis por adequada atenção à mulher na gestação e redução das mortes em virtude de causas reduzíveis por adequada atenção à mulher no parto e ao recém-nascido e por ações de promoção à saúde 17 .

Já os óbitos evitáveis por ações adequadas de diagnóstico e tratamento, que apresentaram redução no país, tiveram um aumento de cerca de $70 \%$ em Pelotas no mesmo período, ficando o coeficiente de mortalidade acima do nacional para essas causas $(1,65$ por mil nascidos vivos em Pelotas em 2004, contra 1,35 por mil nascidos vivos no Brasil em 2007).

Entre 1993 e 2004, houve um aumento de 27,5\% no número de recém-nascidos que apresentaram complicações neonatais, um marcador de risco de mortalidade neonatal. Cerca da metade desses recém-nascidos (45,9\%) era pré-termo, a maioria $(86,1 \%)$ necessitou de hospitalização em unidade de tratamento intensivo e $14,6 \%$ vieram a falecer. Em 1993, 38 (47\%) dos oitenta óbitos evitáveis foram de pré-termos, enquanto, em 2004, essa proporção foi $30 \%$ maior (39 de 65). A sobrevida de pré-termos com idade gestacional ao nascer inferior a 34 semanas aumentou entre 1993 e 2004, o que indica a melhoria da qualidade do cuidado prestado pelas unidades de cuidados intensivos disponibilizados na cidade. Particularmente, a incidência de óbitos evitáveis entre os nascidos com 32-33 semanas de idade gestacional caiu à metade em 2004, comparativamente a $1993(6,2 \%$ contra $13,3 \%$, respectivamente, em 2004 e 1993). No entanto, redução semelhante não se observou entre os pré-termos limítrofes (34-36 semanas), os quais, em 2004, corresponderam a dois terços de todos os pré-termos de Pelotas 21, ou entre os nascidos com 37 semanas ou mais.

A despeito da ampla oferta, utilização e cobertura da atenção pré-natal e independentemente de os nascimentos ocorrerem quase que exclusivamente em ambiente hospitalar, o número de óbitos infantis por causas que seriam evitáveis com os atuais recursos do SUS é muito alto em Pelotas. As desvantagens socioeconômicas e biológicas das mães de 2004, em comparação com as de 1993, associadas ao aumento dos nascimentos de pré-termos, possivelmente tenham contribuído para a persistência das taxas de óbitos evitáveis observada no período. Intervenções para combater a inversa e significante associação entre nível socioeconômico e mortalidade infantil, que no Brasil se expressa tanto entre regiões, como no âmbito dos estados e municípios, continuarão sendo um desafio no combate às iniquidades. A necessidade de manter um foco permanente na saúde infantil permanece nos dias atuais. 


\section{Resumen}

La mortalidad infantil evitable (por acciones adecuadas de inmunización, cuidados maternos durante el embarazo y parto, cuidados a la salud del recién nacido, diagnóstico y tratamiento, y acciones de promoción de salud) fue comparada en las cohortes de nacimiento de Pelotas, Río Grande do Sul, Brasil, de 1993 y 2004. Las muertes fueron monitorizadas mediante visitas a hospitales, oficinas de registro de nacimientos y muertes, cementerios y Delegaciones de Salud Regionales, asi como mediante una búsqueda en el banco de datos de muertes ocurridas en el país. Se registraron 5.249 nacidos vivos con 111 muertes infantiles en la cohorte de 1993 y 4.231 nacidos vivos con 82 muertes infantiles en 2004. El coeficiente de mortalidad infantil evitable fue 15,2:1.000 nacidos vivos en 1993 y 15,4 en 2004. Los coeficientes de mortalidad neonatal y post-neonatal evitable fueron, respectivamente, 11,2 y 4,0, en 1993 y 10,9 y 4,5 en 2004. El nacimiento prematuro fue la principal variable asociada con mortalidad evitable en ambas cohortes. Estrategias para prevenir el nacimiento de prematuros podrá ayudar a reducir la mortalidad infantil en la ciudad.

Mortalidad Infantil; Causas de Muerte; Estudios de Cohortes

\section{Colaboradores}

I. S. Santos concebeu o projeto, participou na interpretação dos dados e redigiu a primeira versão do manuscrito. A. Matijasevich realizou a análise dos dados e contribuiu na interpretação dos resultados e na redação da primeira versão do manuscrito. L. R. M. R. Gorgot e N. C. J. Valle participaram da análise e contribuíram na interpretação dos dados. A. M. Menezes colaborou na interpretação dos dados e na revisão crítica do conteúdo intelectual do manuscrito.

\section{Agradecimentos}

As coortes de nascimentos de Pelotas de 1993 e 2004 , conduzidas pelo Programa de Pós-graduação em Epidemiologia da Universidade Federal de Pelotas, são atualmente financiadas pelo The Wellcome Trust, UK. Fases anteriores foram financiadas pela União Européia Organização Mundial da Saúde, Programa de Apoio a Núcleos de Excelência (PRONEX), CNPq, Ministério da Saúde e Pastoral da Criança. As análises deste artigo foram realizadas com recursos provenientes do Departamento de Análise de Situação em Saúde (DASIS) do Ministério da Saúde. I. S. Santos, A. Matijasevich e A. M. Menezes são bolsistas de produtividade científica do CNPq.

\section{Referências}

1. Instituto Brasileiro de Geografia e Estatística. Observações sobre a evolução da mortalidade no Brasil: o passado, o presente e perspectivas. Rio de Janeiro: Instituto Brasileiro de Geografia e Estatística; 2010.

2. Bhutta ZA, Black RE. Global maternal, newborn, and child health: so near and yet so far. N Engl J Med 2013; 369:2226-35.

3. Departamento de Análise de Situação em Saúde, Secretaria de Vigilância em Saúde, Ministério da Saúde. Saúde Brasil 2012: uma análise da situação de saúde e dos 40 anos do Programa Nacional de Imunizações. Brasília: Ministério da Saúde; 2013.
4. Santos IS, Menezes AMB, Mota DM, Albernaz EP, Barros AJD, Matijasevich A, et al. Infant mortality in three population-based cohorts in Southern Brazil: trends and differentials. Cad Saúde Pública 2008; 24 Suppl 3:S451-60.

5. Santos IS, Matijasevich A, Barros AJD, Albernaz EP, Domingues MR, Valle NC, et al. Avoidable deaths in the first four years of life among children in the 2004 Pelotas (Brazil) birth cohort study. Cad Saúde Pública 2011; 27 Suppl 2:S185-97.

6. Gorgot LRM, Santos I, Valle N, Matijasevich A, Barros AJ, Albernaz E. Óbitos evitáveis até 48 meses de idade entre as crianças da coorte de nascimentos de Pelotas de 2004. Rev Saúde Pública 2011; 45:334-42. 
7. Barros AJD, Santos IS, Matijasevich A, Araújo CL, Gigante DP, Menezes AMB, et al. Methods used in the 1982, 1993, and 2004 birth cohort studies from Pelotas, Rio Grande do Sul State, Brazil, and a description of the socioeconomic conditions of participants' families. Cad Saúde Pública 2008; 24 Suppl 3:S371-80.

8. Puffer RR, Serrano CV. Patterns of mortality in childhood: the inter-american investigation of mortality in childhood. Washington DC: PanAmerican Health Organization; 1973.

9. World Health Organization. International statistical classification of diseases and related health problems. 9th Rev. Geneva: World Health Organization; 1982

10. World Health Organization. International statistical classification of diseases and related health problems. 10th Rev. Geneva: World Health Organization; 1993.

11. Malta DC, Duarte EC, Almeida MF, Dias MAS, Morais Neto OL, Moura L, et al. Lista de causas de mortes evitáveis por intervenções do Sistema Único de Saúde do Brasil. Epidemiol Serv Saúde 2007; 16:233-44

12. Martin JA, Hamilton BE, Sutton PD, Ventura SJ, Menacker F, Munson ML. Births: final data for 2003. Natl Vital Stat Rep 2005; 54:1-116.

13. Fenton TR. A new growth chart for preterm babies: Babson and Benda's chart updated with recent data and a new format. BMC Pediatr 2003; 3:13.

14. Dubowitz LM, Dubowitz V, Goldberg C. Clinical assessment of gestational age in the newborn infant. J Pediatr 1970; 77:1-10.

15. Williams RL, Creasy RK, Cunningham GC, Hawes WE, Norris FD, Tashiro M. Fetal growth and perinatal viability in California. Obstet Gynecol 1982; 59:624-32.
16. Victora CG, Huttly SR, Fuchs SC, Olinto MT. The role of conceptual frameworks in epidemiological analysis: a hierarchical approach. Int J Epidemiol 1997; 26:224-7.

17. Malta DC, Duarte EC, Escalante JJC, Almeida MF, Sardinha LMV, Macário EM, et al. Mortes evitáveis em menores de um ano, Brasil, 1997 a 2006: contribuições para a avaliação de desempenho do Sistema Único de Saúde. Cad Saúde Pública 2010; 26: 481-91.

18. Victora CG, Aquino EM, Leal MC, Monteiro CA, Barros FC, Szwarcwald CL. Maternal and child health in Brazil: progress and challenges. Lancet 2011; 377:1863-76.

19. Kim D, Saada A. The social determinants of infant mortality and birth outcomes in Western developed nations: a cross-country systematic review. Int J Environ Res Public Health 2013; 10:2296-335.

20. O'Hare B, Makuta I, Chiwaula L, Bar-Zeev N. Income and child mortality in developing countries: a systematic review and meta-analysis. J R Soc Med 2013; 106:408-14.

21. Santos IS, Matijasevich A, Silveira MF, Sclowitz IK, Barros AJD, Victora CG, et al. Associated factors and consequences of late preterm births: results from the 2004 Pelotas birth cohort. Paediatr Perinat Epidemiol 2008; 22:350-9.

Recebido em 11/Jul/2013

Versão final reapresentada em 11/Jan/2014

Aprovado em 01/Abr/2014 